\title{
Wandel im Bereich der Luftrettung Die Rolle des Rettungshubschraubers Christoph 18 in der Notfallrettung
}

\author{
P. Sefrin, H. Kuhnigk, H. Lohs \\ Klinik für Anaesthesiologie der Universitätsklinik Würzburg (Direktor: Prof. Dr. med. N. Roewer)
}

NOTFALLMEDIZIN 2003; 29: 98-106

Der Stellenwert von Rettungshubschraubern (RTH) im Rettungssystem Deutschlands steht nach wie vor aus finanziellen Gründen zur Diskussion. Gegenüber dem bodengebundenen Rettungsdienst besitzt der RTH Vorteile, die besonders bei weiter entfernt liegenden Einsatzorten zum Tragen kommen. Dem Einsatz sind aber Grenzen gesetzt, weshalb er nur als additives System wirken kann. Von einem anfänglich auf Verkehrsunfälle und traumatologische Notfälle spezialisierten Rettungsmittel ist der RTH mit seiner Besatzung zu einem universellen Versorgungsinstrument geworden. Als Diskussionsbeitrag sollen deshalb Einsatzdaten des RTH Christoph 18 vorgestellt werden.

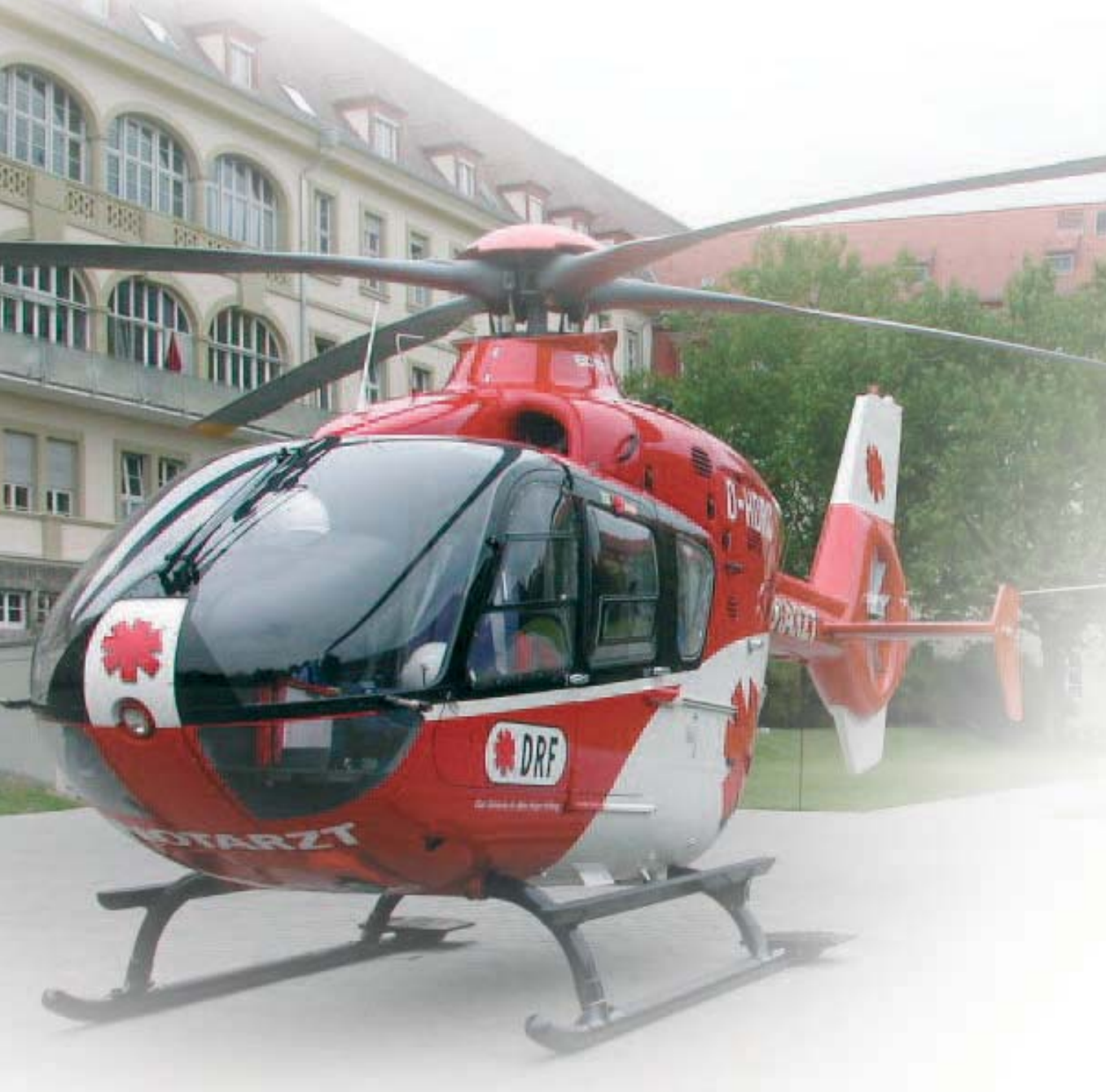

D er Stellenwert von Rettungshubschraubern (RTH) im Rettungssystem Deutschlands steht nach wie vor zur Diskussion. Für eine objektives Urteil über die Bedeutung und eine Bewertung hinsichtlich der Effektivität und Effizienz fehlen nach Ansicht der Konsensusgruppe „Luftrettung“ des Ausschusses Rettungswesen medizinische und ökonomische Datengrundlagen (13).

Da eine Stationierung eines Rettungshubschraubers in Würzburg mit direkter Anbindung an das Universitätsklinikum aus städtebaulichen und immissionsschutzrechtlichen Gründen scheiterte, wurde 1981 das Kreiskrankhaus Ochsenfurt als Standort für die Luftrettung in Unterfranken gewählt. Ausschlaggebend war die günstige Lage zu den Unfallschwerpunkten an den vier Autobahnen (BAB A3, A7, A70, A81) sowie die Höhenlagen des Krankenhauses mit einer hindernisfreien An- und Abflugmöglichkeit über unbewohntes Gebiet. Nachdem die Luftrettung im Rettungsdienst heute teilweise in Gesetze implementiert ist, erhebt sich auch für den Würzburger Raum die Frage nach dem Beitrag des in diesem Bereich stationierten Luftrettungsmittels Christoph 18.

Die Luftrettung ist nach geltender Rechtsauffassung als Bestandteil der allgemeinen Gesundheitsfürsorge eine öffentliche Aufgabe. Sie unterliegt als Teil des Rettungsdienstes der Regelungskompetenz der Länder, hier dem Bayerischen Rettungsdienstgesetz (BayRDG). Die Einsätze werden von der Rettungsleitstelle Würzburg geführt, die Einsatzkosten übernehmen die Träger der Sozialversicherung. 


\section{Luftrettung als \\ additives System}

Der Rettungshubschrauber in Ochsenfurt wird bezüglich des Rettungsdienstpersonals vom Bayerischen Roten Kreuz und dem Malteser Hilfsdienst, bei den Ärzten vornehmlich von Anaesthesisten des Kreiskrankenhauses Ochsenfurt und der Klinik für Anaesthesiologie der Universitätsklinik Würzburg besetzt. Die Gestellung der Maschinen und Piloten hat im Berichtzeitraum mehrfach gewechselt zwischen dem Bundesgrenzschutz, dem ADAC und der Deutschen Rettungsflugwacht. Der Einsatzbereich umfasst nicht nur den Rettungsdienstbereich Würzburg, sondern auch Teile von Oberund Mittelfranken und den nördlichen Bereich von Baden-Württemberg. Gegenüber dem bodengebundenen Rettungsdienst besitzt der RTH einige Vorteile, die besonders bei weiter entfernt liegenden Einsatzorten zum Tragen kommen, wie z.B. Verkürzung des therapiefreien Intervalls durch Unabhängigkeit von der Verkehrslage, Abdeckung größerer Gebiete mit notärztlicher Leistung, schonender Patiententransport, Optimierung der Krankenhauszuweisung und andere. Dem Einsatz sind aber auch technische und fliegerische Grenzen gesetzt, weshalb er nur als additives System wirken kann. Aus diesen Gründen sollte das Einsatzspektrum und der Zustand der Patienten im Sinne der Effektivität vor dem Hintergrund der Kosten analysiert werden.

\section{Analyse aller Rettungs- \\ hubschrauber-Einsätze}

In dem Zeitraum vom 31.07.1980 bis 31.12.1995 wurden retrospektiv alle RTH-Einsätze analysiert. Als Datenquelle dienten das Bordbuch von Christoph 18 (Ch 18) sowie die Einsatzprotokolle. Mit Hilfe eines Datenerhebungsbogens wurden die Einsatzdaten anonymisiert, auf einem PC erfasst und mit dem System „PCMedas“ des Rechenzentrums der Universität Würzburg ausgewertet. Die Ergebnisdarstellung erfolgte mit dem Textverarbeitungsprogramm „Word“ sowie dem Tabellenkalkulationsprogramm „Excel“ (Microsoft Seattle, USA).
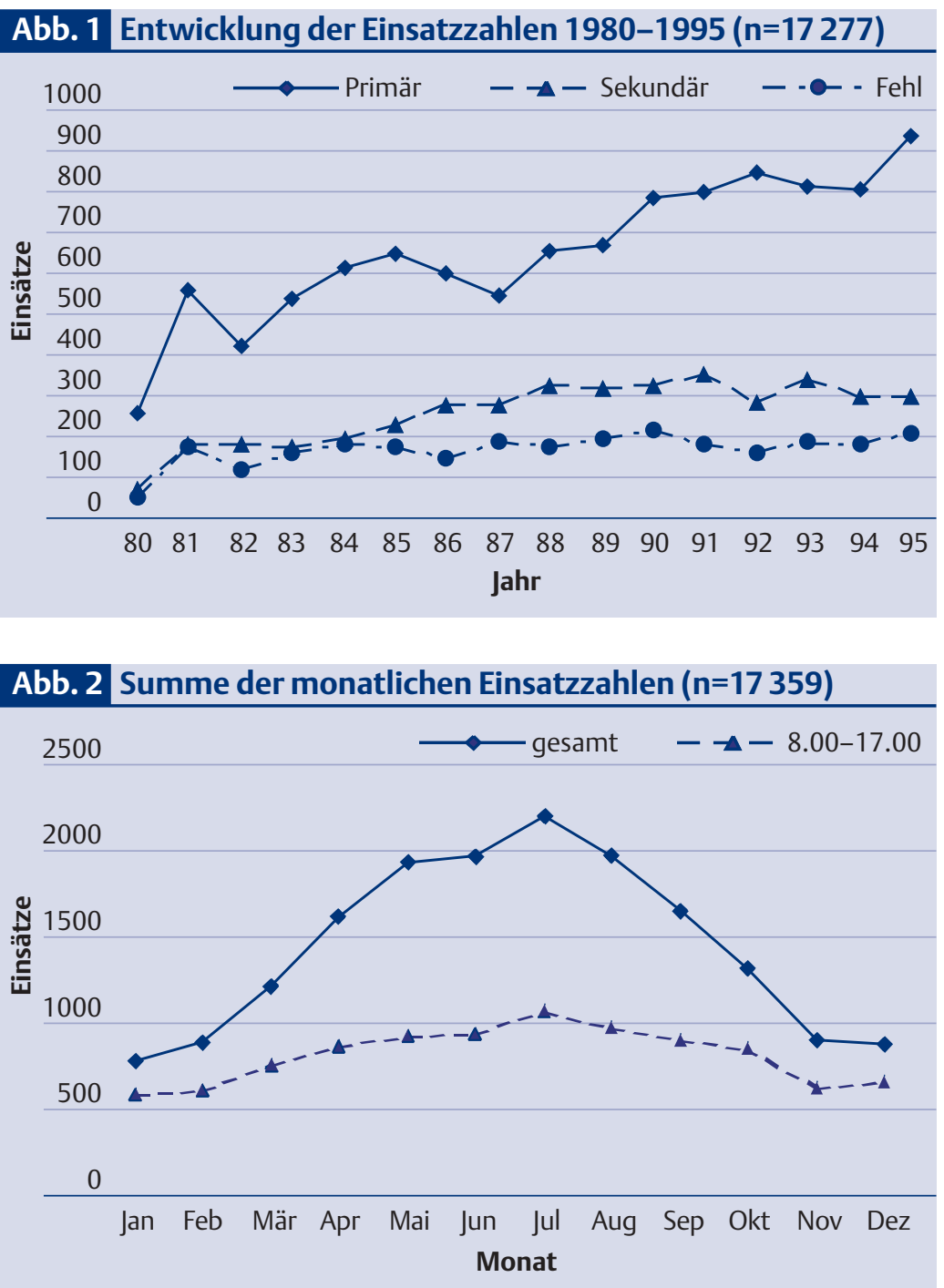

Es folgte eine Grundaufzählung sowie eine deskriptive Darstellung aller Beobachtungen. Aus der differenten Verfügbarkeit einzelner Variablen (Dokumentationsmoral) resultieren variable Grundgesamtheiten. Für quantitative Merkmale (Maße) wurden Mittelwert, Standardabweichung und Spannweite (auf Minimum - Maximum) ermittelt, für die qualitativen Merkmale (Klassen) die absolute und relative Häufigkeitsverteilung. Qualitative Merkmale wurden zur Analyse von Zusammenhängen in Kreuztabellen verknüpft und eine Kreuzauszählung sowie der ChiQuadrat Test durchgeführt.

\section{Ergebnisse}

Entwicklung der Einsätze

Die Gesamtzahl der Einsätze von 1980 bis 1995 betrug 17277
(Abb. 1), wobei eine kontinuierliche jährliche Steigerung festzustellen war. 60,5\% der Einsätze waren Primär- und 23,6\% Sekundäreinsätze. Der Anteil der Fehleinsätze betrug 15\%. Insgesamt wurden $15328 \mathrm{~Pa}$ tienten versorgt. Transportiert wurden dagegen nur 5333 Patienten. Vier Patienten sind während des Transportes verstorben. $4097 \mathrm{~Pa}$ tienten wurden mit dem Rettungswagen (RTW) nach Erstversorgung transportiert, wobei bei 1066 Fällen der Notarzt den bodengebundenen Transport begleitete.

Einsatzhäufigkeit nach Monaten

Die Einsatzhäufigkeit wies für die gesamte Beobachtungszeit deutliche jahreszeitliche Schwankungen auf, wobei $65,4 \%$ der Einsätze in die Monate April bis September fielen 


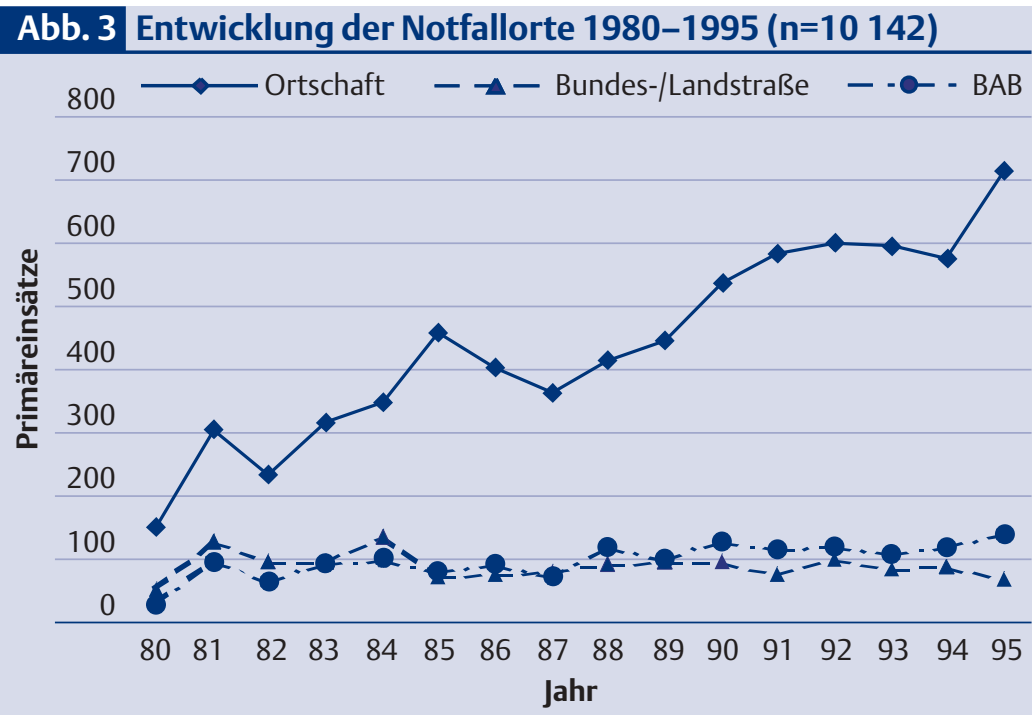

Abb. 4 Entwicklung der Notfallkategorien 1980-1995 ( $n=10455)$

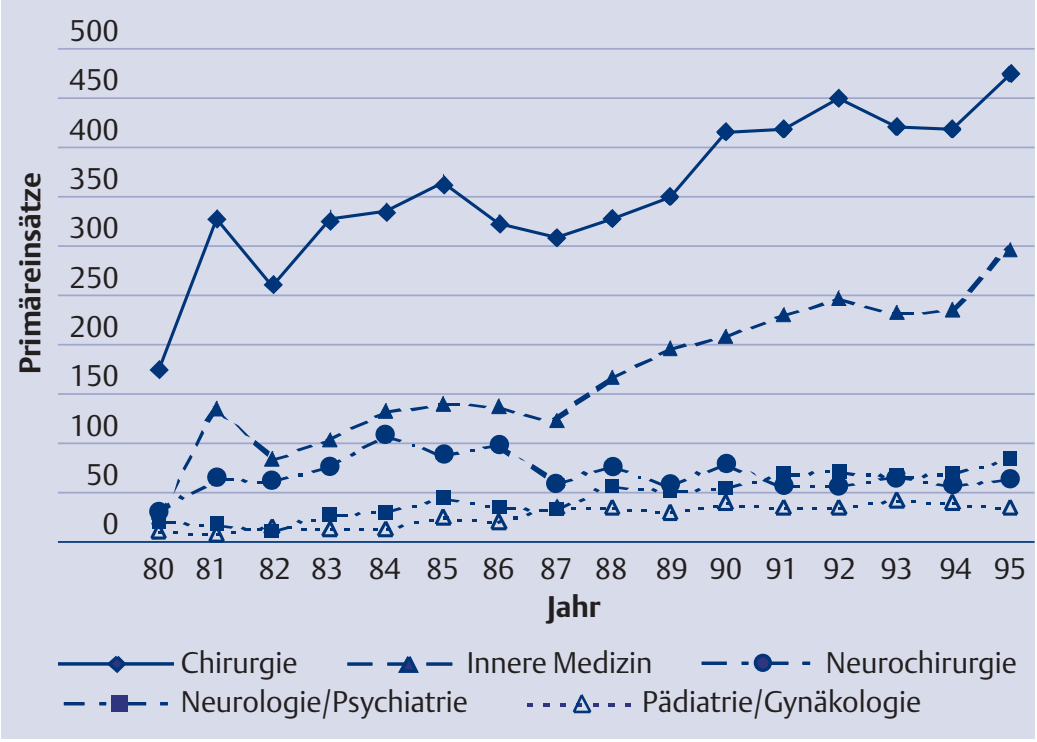

\section{Abb. 5 Entwicklung der Diagnosegruppen relativ 1980-1995 $(n=14131)$}

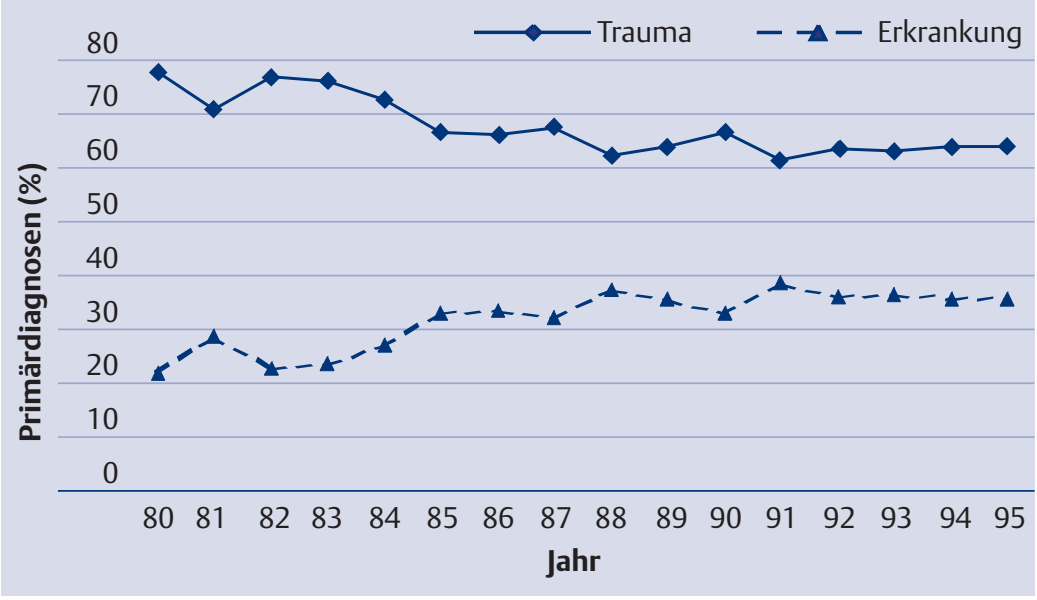

(Abb. 2). Die geringste Einsatzhäufigkeit entfiel auf den Monat Januar. Der Einsatzgipfel im Juli resultierte aus dem hohen Anteil von Primäreinsätzen in diesem Monat (67\%).

\section{Einsatzzeit}

Der RTH zeichnet sich durch sein schnelles Eintreffen am Notfallort aus. Im Durchschnitt dauerte der RTH-Einsatz 56,1 \pm 27,7 Minuten. Um den Notfallort zu erreichen, benötigte der RTH durchschnittlich $11,5 \pm 5,9$ Minuten.

\section{Notfallorte der Primäreinsätze}

Einsätze innerhalb von Ortschaften haben von $1981(\mathrm{n}=306)$ bis 1995 $(\mathrm{n}=713)$ um 57,1\% zugenommen, während Einsätze auf Autobahnen in der gleichen Zeit nur eine Steigerung um 31,7\% (von 97 auf 142) aufwiesen (Abb. 3). Im Gegensatz dazu verringerten sich die Einsätze auf Bundes- und Landstraßen um 43,8\% (von 128 auf 72 ).

\section{Notfallkategorien}

Mehr als die Hälfte der Primäreinsätze galt traumatologischen Notfällen. Während die absoluten Zahlen - mit Ausnahme der Jahre 1982, 1986, 1987 und 1993/94 - kontinuierlich stiegen, war ihr Anteil am Primäreinsatzaufkommen mit 61,9\% im Jahre 1982 am höchsten. Seit dieser Zeit ist dieser Anteil rückläufig und betrug 1995 nur noch 50,4\%.

70\% der Unfälle waren Verkehrsunfälle, gefolgt von $11,4 \%$ Arbeitsunfällen. Der Anteil der Verkehrsunfälle am Gesamteinsatzaufkommen nahm über die Jahre kontinuierlich ab.

Die neurochirurgischen Notfälle stiegen nur bis 1984, um danach abzufallen (Abb. 4). Jeder vierte Einsatz galt einem internistischen Notfall. Bis auf die Jahre 1982, 1986/87 und 1993/94 stiegen die Absolutzahlen der internistischen Notfälle jährlich an. Ihr Anteil am Einsatzaufkommen stieg von $18,8 \%$ auf $31,2 \%$.

\section{Primärdiagnose}

Im Vordergrund der Schädigungen waren mit 63,5\% die Folgen von Traumen (Abb. 5). Bei 32,1\% waren akute Erkrankungen der Anlass zum Einsatz des RTHs, wobei bei beiden Diagnosegruppen eine Angleichung 
der Einsätze über die Jahre hinweg von anfänglich $80 \%$ Trauma und 20\% Erkrankungen zu verzeichnen war.

\section{Einzeldiagnosen}

Bei der Verteilung der verletzten Körperregionen (Abb. 6) lag das Schädelhirntrauma über die Jahre hinweg mit 22,5\% vor den Verletzungen der Extremitäten $(17,4 \%)$ und der Weichteile (15,6\%). Andere Verletzungen waren mit weniger als $10 \%$ am Gesamtaufkommen beteiligt (Thoraxtrauma 8,6\%, Wirbelsäulenverletzungen $7,9 \%$, Polytrauma $5,4 \%$, Bauch-

trauma 4,9\%). Bei der Analyse der Verteilung dieser Schädigungen ist eine fast gleichsinnige Entwicklung über die Jahre hinweg zu beobachten.

Die größte Erkrankungsgruppe bildeten die Störungen des kardiozirkulatorischen Systems (18,7\%), gefolgt von Erkrankungen des Zentralen Nervensystems (10,1\%). Unter 10\% liegen die Erkrankungen des respiratorischen Systems (4,7\%), Intoxikationen (2,7\%), Störungen des Stoffwechsels und des Endokriniums $(1,4 \%)$ und sonstige. Auch bei den Erkrankungen war über die Jahre hinweg eine kontinuierliche absolute Steigerung zu beobachten.

\section{Schweregrade}

Entsprechend der NACA-Skala (Abb. 7, Tab. 1) waren 19,3\% der Fälle als gering geschädigt einzustufen (NACA I und II). Fast ein Drittel der Patienten war mittelschwer geschädigt (31,2\%). Schwer verletzt oder erkrankt (NACA IV und V) waren 39,6\% der versorgten Patienten. Reanimationsmaßnahmen (NACA VI) waren bei 5,8\% der Patienten notwendig, während bei 4,1\% bei Eintreffen am Notfallort der Tod festgestellt wurde.

Bei einem Vergleich der NACASchweregrade bei den Traumapatienten und Patienten mit akuten Erkrankungen waren keine einheitlichen Tendenzen erkennbar. Während der Anteil der leichten Schädigungen (NACA I-III) bei Erkrankungen 46,3\% betrug, war der Anteil bei den traumatologischen Patienten 57\%. Bei den traumatologischen Patienten war bei 6,5\% der Patienten eine Reanimation im Gegensatz zu 3,4\% bei den Erkrankungen notwendig, während bei 9,5\% der Patienten akut durch eine Krankheit und nur bei 2,4\% durch einen Unfall der Tod eingetreten war.

\section{Transportmittel}

Bei jedem zweiten traumatologischen Notfall ( $n=2278)$ wurde der Patient - nach Versorgung durch den RTH-Arzt - auch per Hubschrauber ins Krankenhaus geflogen. In 30,2\% wurde der Patient ohne RTH-Arzt
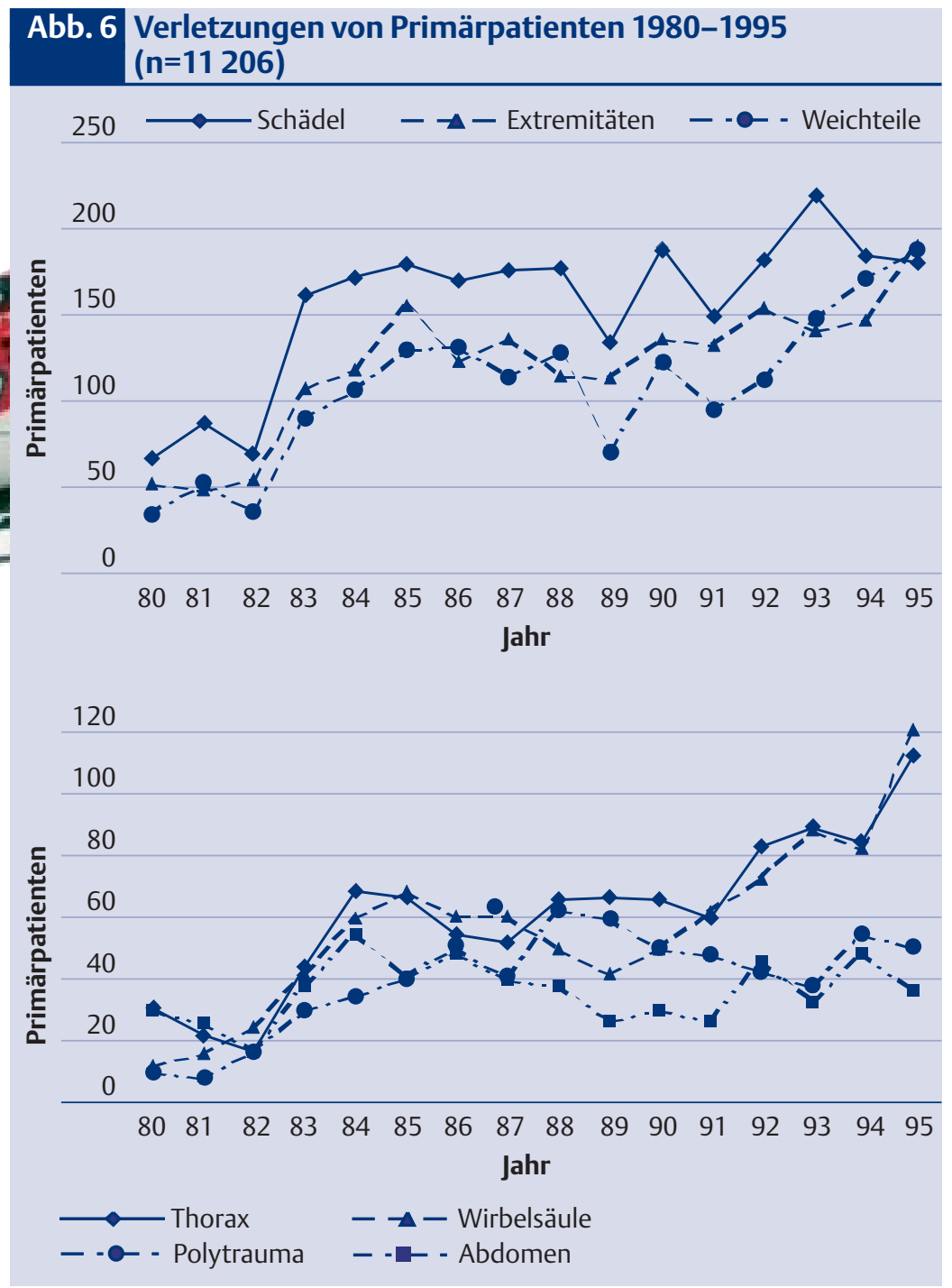

\section{Abb. 7 Verteilung der NACA-Schweregrade $(n=2486)$}

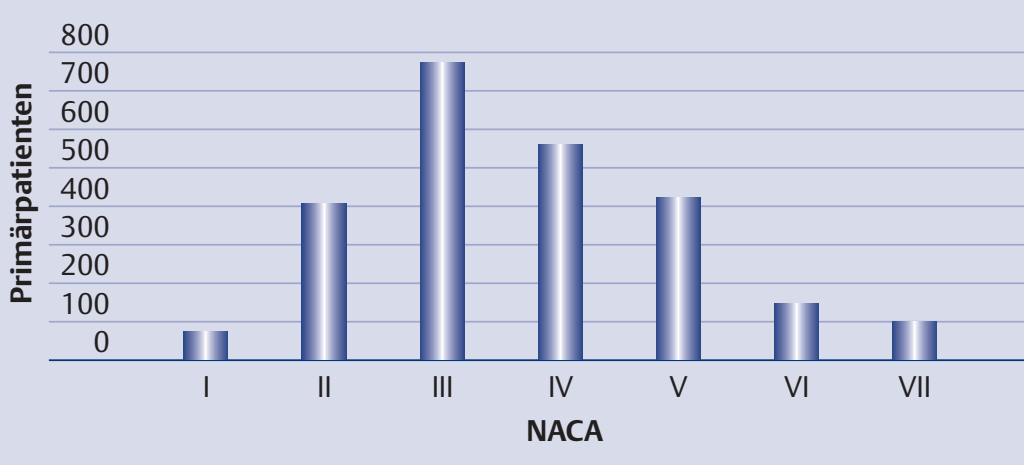




\section{Tab. 1 Häufigkeit der NACA-Schweregrade in Abhängigkeit von der Notfallkategorie $(n=2486)$}

\begin{tabular}{|c|c|c|c|c|c|}
\hline NACA & $\begin{array}{l}\text { Chirurgischer } \\
\text { Notfall* }^{*}(\%) \\
n=1277\end{array}$ & $\begin{array}{l}\text { Internistischer } \\
\text { Notfall n (\%) } \\
n=739\end{array}$ & $\begin{array}{l}\text { Neurochirurg. } \\
\text { Notfall } n(\%) \\
n=170\end{array}$ & $\begin{array}{l}\text { Neurol./Psych. } \\
\text { Notfall } n(\%) \\
n=198\end{array}$ & $\begin{array}{l}\text { Pädiatr./Gyn. } \\
\text { Notfall n (\%) } \\
n=102\end{array}$ \\
\hline I & $41(3,2)$ & $23(3,1)$ & - & $7(3,5)$ & $3(2,9)$ \\
\hline II & $242(19,0)$ & $116(15,7)$ & $6(3,5)$ & $24(12,1)$ & $18(17,6)$ \\
\hline III & $444(34,8)$ & $203(27,5)$ & $23(13,5)$ & $68(34,3)$ & $37(36,3)$ \\
\hline IV & $262(20,5)$ & $184(24,9)$ & $43(25,3)$ & $50(25,2)$ & $29(28,4)$ \\
\hline V & $175(13,7)$ & $118(16,0)$ & $73(42,9)$ & $42(21,2)$ & $9(8,8)$ \\
\hline VI & $83(6,5)$ & $25(3,4)$ & $25(14,7)$ & $7(3,5)$ & $4(3,9)$ \\
\hline VII & $30(2,4)$ & $70(9,5)$ & - & - & $2(2,0)$ \\
\hline Mittel & 3,5 & 3,8 & 4,5 & 3,6 & 3,4 \\
\hline
\end{tabular}

\section{Tab. 2 Transportmittel in Abhängigkeit von der Notfallkategorie ( $n=10$ 432)}

\begin{tabular}{llllll} 
Notfallkategorie & $\begin{array}{l}\text { RTH } \\
\mathbf{n ( \% )}\end{array}$ & $\begin{array}{l}\text { RTW } \\
\mathbf{n ( \% )}\end{array}$ & $\begin{array}{l}\text { kein } \\
\text { Transport } \\
\mathbf{n}(\%)\end{array}$ & $\begin{array}{l}\text { RTW + } \\
\text { RTH-Arzt } \\
\mathbf{n}(\%)\end{array}$ & $\begin{array}{l}\text { 1. and. RM } \\
\text { + 2. RTH } \\
\mathbf{n}(\%)\end{array}$ \\
\hline Chirurgischer Notfall* & $2878(50,9)$ & $1707(30,2)$ & $399(7,1)$ & $329(5,8)$ & $342(6,0)$ \\
Internistischer Notfall & $541(20,4)$ & $869(32,8)$ & $672(25,4)$ & $501(18,9)$ & $66(2,5)$ \\
Neurochirurg. Notfall & $711(68,0)$ & $108(10,3)$ & $19(1,8)$ & $37(3,5)$ & $171(16,4)$ \\
Neurolog./Psych. Notfall & $203(29,1)$ & $260(37,2)$ & $66(9,5)$ & $127(18,2)$ & $42(6,0)$ \\
Pädiatr./Gynäkol. Notfall & $179(46,6)$ & $82(21,4)$ & $31(8,1)$ & $70(18,2)$ & $22(5,7)$ \\
*einschließlich HNO-/Augenheilkunde, Zahn-/Mund-/Kieferchirurgie & &
\end{tabular}

mit einem RTW ins Krankenhaus transportiert ( $\mathrm{n}=1707$ ), nur in $5,8 \%$ begleitete der RTH-Arzt im bodengebundenen Rettungsmittel. In 7,1\% $(n=399)$ wurde kein Transport durchgeführt. In 6\% wurde der Patient von anderen Rettungsmitteln übernommen (Tab. 2).

Neurochirurgische Patienten wurden häufiger (68\%) und internistische Patienten wesentlich seltener (20,4\%) mit dem Hubschrauber transportiert. Am häufigsten wurden internistische Patienten nicht transportiert (25,4\%), dagegen erfolgte ein RTW-Transport häufiger bei internistischen (32,8\%) als bei neurochirurgischen Patienten (10,3\%).

Mit zunehmender Schwere der Verletzungen bzw. Erkrankungen stieg auch der Anteil der primär mit dem RTH transportierten Patienten (Tab. 3). Während Patienten mit NACA I und II in 16,2 bzw. 22,9\% mit dem RTH transportiert wurden, lag der Anteil beim Schweregrad III bereits bei $35,2 \%$, um bis zum Schweregrad VI auf 68,3\% anzusteigen.

\section{Reanimationen}

Bei 382 Einsätzen wurde eine kardiopulmonale Reanimation (cardiopulmonary resuscitation - CRP) durchgeführt, die in $28,5 \%$ der Fälle primär erfolgreich verlief (Tab. 4). In 273 Fällen (71,5\%) mussten die Wiederbelebungsversuche erfolglos abgebrochen werden. Die meisten Reanimationen ( $n=261$ ) wurden bei internistischen Patienten durchgeführt, wovon $29,5 \%$ primär erfolgreich waren. Für 84 Wiederbelebungsversuche bei traumatologischen Patienten war eine primäre Erfolgsquote von 19\% zu verzeichnen. Die höchste primäre Erfolgsquote wurde bei der geringen Zahl von Reanimationen bei pädiatrischen und gynäkologischen Notfallpatienten ( $n=23$ ) mit 43,5\% erreicht.

\section{Diskussion der Einsatzdaten}

Der medizinische Notfall impliziert die akute Erkrankung, eine akute Gefährdung oder die Möglichkeit einer akuten Gefährdung der Gesundheit (13). Medizinische Maß- nahmen zur Abwehr möglicher vorübergehender oder bleibender Schäden sind dem Betroffenen so schnell wie möglich zukommen zu lassen, denn die Akutversorgung kann nur erfolgreich sein, wenn sie unmittelbar nach der Schädigung durchgeführt werden kann. Damit kommt der frühen Notfallversorgung eine - wenn nicht die - entscheidende Rolle bei der Wiederherstellung und Stabilisierung von Notfallpatienten zu. Je kürzer die Zeit bis zum Eintreffen am Notfallort, desto größer ist die Chance der Wiederherstellung. Bei einer Dauer von fünf Minuten lag der Anteil der Patienten, die mehr als 20 Tage im Krankenhaus verbleiben mussten, nur bei $28 \%$, während bei Eintreffzeiten von mehr als 15 Minuten dieser Anteil 45,5\% und bei einem Zeitraum von länger als 20 Minuten sogar $61 \%$ betrug. Diese Abhängigkeit betrifft sowohl die Intensivstation- wie auch die Krankenhausverweilzeit (23). Die Bedeutung des Rettungshubschraubers im gesamten Rettungssystem resultiert aus seiner Fähigkeit, den Patienten nicht nur in dieser zeitkritischen Phase frühzeitig $\mathrm{zu}$ erreichen, sondern auch die notfallmedizinische Versorgung durch einen qualifizierten Notarzt garantieren zu können.

\section{Gestiegene Akzeptanz der Luftrettung}

Im Rettungsdienst in Deutschland ist in den zurückliegenden Jahren generell von steigenden Einsatzzahlen auszugehen. So stieg das gesamte Einsatzaufkommen von 5,97 Millionen im Jahre 1985 auf 9,04 Millionen in den Jahren 1996/97 (=+51,4\%) (24). Diese Steigerung verteilt sich sowohl auf den bodengebundenen Rettungsdienst, wie auch auf die Luftrettung als obligaten Bestandteil des gesamten Rettungssystems. Die Luftrettung ist als additives Rettungssystem im Bereich der Notfallrettung einzustufen, dessen Anteil am Gesamteinsatzaufkommen der ärztlichen Einsätze 4\% (20) betrug - was 199657000 Einsätze entsprach. Daher erscheint eine Analyse des Standortes eines Rettungshubschraubers mit einem Jahresmittel der Einsätze von 1126 
Einsätzen/Jahr

(über einen 15-Jahres-

Zeitraum) einen reprä-

sentativen Überblick geben

zu können. Die bundes-

einheitliche Steigerung der Einsatzquoten lässt sich auch - sowohl im Bereich der Primär- wie Sekundäreinsätze - tendenziell am Standort von Christoph 18 nachvollziehen. Diese Steigerung ist einmal auf eine erhöhte Akzeptanz dieses Rettungsmittels, aber auch auf seine Funktion als Rückfallebene bei der NichtVerfügbarkeit des Bodennotarztes zurückzuführen. Der Einsatzbereich des RTH Ch 18 deckt in großen Teilen einen ländlichen Bereich mit teils längeren Anfahrtswegen des bodengebundenen Notarztes ab.

\section{Insgesamt mehr Primär- \\ als Sekundäreinsätze}

Bei der Trennung in Primär- und Sekundäreinsätze stiegen die Einsätze über die Jahre um 67,7\% für die Primär- und 63,2\% für die Sekundäreinsätze (Tab. 5). Im Mittel entfielen $60,5 \%$ aller Einsätze auf Primär- und 23,6\% auf Sekudäreinsätze und liegen damit in Übereinstimmung mit anderen Standorten (1, 9). Für alle deutschen RTH-Stationen wird eine Quote von 65\% für Primäreinsätze (14) und 14-17\% für Sekundäreinsätze $(16,18)$ angegeben. Bei einem Jahresmittel von 173 Fehleinsätzen lag deren Anteil in Ochsenfurt mit $15,4 \%$ unter dem Bundesdurchschnitt von 16-20\% (13, $15)$, was für eine hohe Dispositionssicherheit der Leitstelle spricht. Damit darf für den RTH Ch 18 - was das Einsatzaufkommen betrifft - unterstellt werden, dass er im Trend der bundesdeutschen Luftrettung liegt und seine Auswertung demnach auch als repräsentativ angesehen werden kann.

Einsatzhäufigkeit auch abhängig von der Einsatzverfügbarkeit

Die Analyse der Einsatzhäufigkeit in den einzelnen Monaten zeigt, dass nahezu 2/3 aller Einsätze in den Sommermonaten geflogen wurden: Im Zeitraum April bis September war eine doppelt so hohe Einsatzfrequenz wie für das Winterhalbjahr festzustellen, was sowohl für die
Primär- als auch für die Sekundäreinsätze zutrifft. Ähnliche Einsatzhäufigkeiten werden von anderen Autoren beschrieben $(8,18)$. Hauptgrund hierfür dürfte die auf die Tageszeit beschränkte Einsatzhäufigkeit des RTH sein, wobei die mittlere Tageslänge im Juni beispielsweise 16,2 Stunden und im Dezember nur 8,3 Stunden beträgt. Diese Einsatzhäufigkeit resultiert damit nicht mehr nur aus der Unfallhäufigkeit in den Sommermonaten, sondern primär aus der längeren Einsatzverfügbarkeit. Wenn die Einsatzbereitschaft auf 24 Stunden verlängert würde - was aus medizinischer Sicht durchaus wünschenswert wäre - würden auch nach Annahme von Puhan (18) diese jahreszeitlichen Schwankungen verringert werden. Eine Egalisierung ist nicht zu erwarten, da neben den Tageszeiten auch Witterungseinflüsse hinzukommen, die im Winter zu einer weitergehenden Behinderung der Einsatzfähigkeit führen. Trotzdem darf die Tatsache nicht außer Acht gelassen werden, dass gerade in
Würzburg, als Knotenpunkt des Urlaubsverkehrs, ein erhöhtes Unfallaufkommen besonders in den Sommermonaten $\mathrm{zu}$ beobachten ist. Dafür spricht auch, dass im Wochenverlauf die Einsatzmaxima am Wochenende liegen, mit einem Schwerpunkt auf den Tagen Freitag und Samstag. Andererseits ist der Rettungshubschrauber an diesen Tagen - durch den Wegfall der hausärztlichen Versorgung und der gemeinsamen Vermittlung des ärztlichen Bereitschafts- und des Notarztdienstes - auch als Rückfallebene bei fehlender bodengebundener Abdeckung - gefragt.

\section{Traumatische Notfälle nehmen} $a b$, internistische steigen an

Im Gegensatz zu den gleich bleibenden Einsatzzahlen für den RTH auf Bundes- und Landstraßen sowie auf Autobahnen, stieg der Anteil der Einsatzorte innerhalb von Ortschaften an, um am Ende 2/3 aller Einsätze auszumachen. Lag der Anteil der innerörtlichen Landeplätze 1981 noch bei 54,6\%, so waren es 1995 über $2 / 3$ bei den Primäreinsätzen. Dies ist durch die deutliche Zunah-

\section{Tab. 3 Transportmittel in Abhängigkeit vom NACA-Schweregrad ( $\mathrm{n=2486)}$}

\begin{tabular}{|c|c|c|c|c|c|}
\hline NACA & $\begin{array}{l}\text { RTH } \\
\text { n (\%) }\end{array}$ & $\begin{array}{l}\text { RTW } \\
\text { n (\%) }\end{array}$ & $\begin{array}{l}\text { kein } \\
\text { Transport } \\
\mathbf{n}(\%)\end{array}$ & $\begin{array}{l}\text { RTW + } \\
\text { RTH-Arzt } \\
\text { n (\%) }\end{array}$ & $\begin{array}{l}\text { 1. and. RM } \\
\text { +2. RTH } \\
\text { n(\%) }\end{array}$ \\
\hline I & $12(16,2)$ & $29(39,2)$ & $28(37,8)$ & $4(5,4)$ & $1(1,4)$ \\
\hline II & $93(22,9)$ & $201(49,5)$ & $60(14,8)$ & $45(11,1)$ & $7(1,7)$ \\
\hline III & $273(35,2)$ & $322(41,6)$ & $44(5,7)$ & $110(14,2)$ & $26(3,4)$ \\
\hline IV & $250(44,0)$ & $112(19,7)$ & $12(2,1)$ & $162(28,5)$ & $32(5,6)$ \\
\hline V & $235(56,4)$ & $32(7,7)$ & $5(1,2)$ & $109(26,1)$ & $36(8,6)$ \\
\hline VI & $99(68,3)$ & $5(3,4)$ & $1(0,7)$ & $28(19,3)$ & $12(8,3)$ \\
\hline VII & - & - & $101(100)$ & - & - \\
\hline
\end{tabular}

\section{Tab. 4 CPR in Abhängigkeit von der Notfallkategorie ( $n=378)$}

\section{Notfallkategorie}

Chirurgischer Notfall*

Internistischer Notfall

Neurochirurg. Notfall

Neurolog./Psych. Notfall

Pädiatr./Gynäkol. Notfall

*einschließlich HNO-/Augenheilkunde, Zahn-/Mund-/Kieferchirurgie
CPR erfolglos abgebrochen n (\%) $68(81,0)$

$184(70,5)$

$3(33,3)$

$1(100)$

$13(56,5)$ 
me des Anteils internistischer Notfälle am Primäreinsatzaufkommen erklärbar. Obwohl die Versorgung akuter Erkrankungen und internistischer Notfälle eine Domäne des bodengebundenen Rettungsdienstes ist, kann Ch 18 - durch den Versorgungsauftrag mit dem Schwerpunkt in der ländlichen Region - gerade bei diesem Patientenkollektiv durch die Schnelligkeit seines Eintreffens ein gleichbleibendes Versorgungsniveau garantieren. Aufgrund der zunehmenden Zahl der Gesamteinsätze ist ein leichter Rückgang der absoluten Anzahl von Notfällen auf Bundes- und Landstraßen zu verzeichnen: Der Anteil ging von 22,9\% im Jahr 1981 auf 7,6\% 1995 zurück. Trotz des im Vergleich zum gesamten Bundesgebiet dichten Straßennetzes - besonders im Bereich der Autobahn im Einzugsgebiet - ist der Einsatzort Straße nicht überrepräsentiert, sondern macht im Jahr 1995 nur noch 1/4 der Primäreinsätze aus. Eine ähnliche Entwicklung ist auch bei anderen Standorten zu beobachten (5), im Gegensatz zum Einsatzort Ludwigshafen, der über Jahre konstant einen Anteil von 50-60\% traumatologischer Einsätze zu verzeichnen hat (13). Generell ist damit eine Zunahme der Einsätze bei akuten Erkrankungen feststell- bar, ohne dadurch den Stellenwert der RTH bei traumatologischen Notfällen zu schmälern.

Diese Beurteilung wird besonders bei der Analyse der Notfallpatienten deutlich. Über die Jahre hinweg waren die traumatologischen Notfälle in mehr als der Hälfte der Einsätze (64,2\%) die häufigste Einsatzindikation, gefolgt von internistischen $(25,4 \%)$ und neurologischen/ psychiatrischen Notfällen (6,7\%). Allerdings konnte festgestellt werden, dass ihr Anteil nach 1984 kontinuierlich (bei steigenden Gesamteinsatzzahlen) zugunsten der internistischen Notfälle abnahm: 1995 betrug ihr Anteil am gesamten Einsatzaufkommen 31,2\%. Für alle bundesdeutschen RTH-Standorte wird ein Anteil von internistischen Notfällen von 34\% angegeben (2).

\section{RTH zunehmend in der}

Akutmedizin akzeptiert

Diese Entwicklung in der Notfallmedizin, die eine deutliche Zunahme neurologischer Notfälle zeigt, ist auch

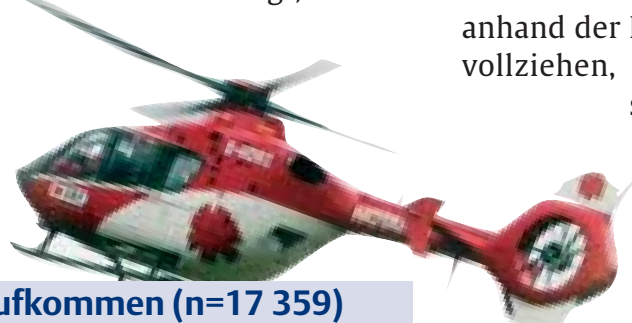

Tab. 5 Anteil der Einsatzarten am Gesamteinsatzaufkommen ( $n=17$ 359)

\begin{tabular}{lllll} 
Jahr & $\begin{array}{l}\text { Primäreinsätze } \\
\mathbf{n}(\%)\end{array}$ & $\begin{array}{l}\text { Sekundäreinsätze } \\
\mathbf{n}(\%)\end{array}$ & $\begin{array}{l}\text { Fehleinsätze } \\
\mathbf{n}(\%)\end{array}$ & $\begin{array}{l}\text { andere Einsätze } \\
\mathbf{n}(\%)\end{array}$ \\
\hline $198 *^{*}$ & $253(66,4)$ & $72(18,9)$ & $51(13,4)$ & $5(1,3)$ \\
1981 & $561(60,9)$ & $182(19,8)$ & $171(18,6)$ & $7(0,8)$ \\
1982 & $421(58,2)$ & $181(25,0)$ & $117(16,2)$ & $4(0,6)$ \\
1983 & $539(61,7)$ & $172(19,7)$ & $161(18,4)$ & $2(0,2)$ \\
1984 & $611(61,6)$ & $193(19,5)$ & $177(17,8)$ & $11(1,1)$ \\
1985 & $649(61,8)$ & $228(21,7)$ & $171(16,3)$ & $2(0,2)$ \\
1986 & $601(58,4)$ & $273(26,5)$ & $143(13,9)$ & $12(1,2)$ \\
1987 & $543(53,7)$ & $278(27,5)$ & $189(18,7)$ & $2(0,2)$ \\
1988 & $652(56,4)$ & $326(28,2)$ & $173(15,0)$ & $5(0,4)$ \\
1989 & $671(56,7)$ & $314(26,5)$ & $194(16,4)$ & $5(0,4)$ \\
1990 & $787(59,0)$ & $326(24,5)$ & $213(16,0)$ & $7(0,5)$ \\
1991 & $802(60,1)$ & $350(26,2)$ & $179(13,4)$ & $3(0,2)$ \\
1992 & $847(65,6)$ & $281(21,8)$ & $159(12,3)$ & $4(0,3)$ \\
1993 & $816(60,6)$ & $338(25,1)$ & $188(14,0)$ & $5(0,4)$ \\
1994 & $809(60,0)$ & $295(23,0)$ & $178(13,9)$ & $2(1,6)$ \\
1995 & $941(65,0)$ & $297(20,5)$ & $204(14,1)$ & $6(0,4)$ \\
Jahresmittel & $681( \pm 142)$ & $266( \pm 62)$ & $173( \pm 24)$ & $6( \pm 3)$ \\
$*$ *inschließlich HNO-/Augenheilkunde, Zahn-/Mund-/Kieferchirurgie & \\
& & & &
\end{tabular}

in der Luftrettung ersichtlich. In einer bayernweiten Auswertung der Notarzteinsätze betrug der Anteil dieser Notfälle am Gesamtkollektiv des Rettungsdienstes 10,2\% (21). Im Gegensatz dazu blieben Einsätze bei Ch 18 im Bereich der Pädiatrie und Gynäkologie anteilig über die Jahre gleich, so dass die steigenden Einsätze bei Erkrankungen im Gesamtkollektiv eindeutig aus zunehmenden internistischen und neurologischen Diagnosen resultiert. Eine derartige Verschiebung im Einsatzspektrum der Luftrettung wird inzwischen auch von anderen Autoren festgestellt, wobei es regionale Differenzen bezüglich des Umfangs gibt $(4,16,22)$. Neben der reellen $\mathrm{Zu}-$ nahme der Notarzt-Einsätze bei internistischen und neurologischen Erkrankungen und abnehmenden Einsätzen bei Verkehrsunfällen, wird das Rettungsmittel Hubschrauber zunehmend in der Notfallmedizin und bei der Disposition der Leitstelle auch im Bereich der Akutmedizin bei Erkrankungen akzeptiert.

Die Entwicklung lässt sich auch anhand der Primärdiagnosen nachvollziehen, wobei Erkrankungen sehr wohl mit einem Trauma kombiniert vorliegen konnte. Dies erklärt auch, dass die Summe beider Fälle die Anzahl der Patienten übersteigen konnte. Bis 1984 lag der Anteil traumatologischer Primärdiagnosen noch über $70 \%$, um sich dann auf $2 / 3$ konstant zu stabilisieren. Die am häufigsten verletzte Körperregion war der Schädel, gefolgt von den Extremitätentraumata. Die Diagnose Polytrauma wurde bei $5,4 \%$ der Patienten gestellt, was bestimmt auch eine Frage der Definition ist. Im Gegensatz dazu war über die Jahre hinweg eine kontinuierliche Zunahme von Erkrankungen zu beobachten, wobei sich der Anteil von 1981 bis 1995 mehr als vervierfacht hat. Diese Steigerung lässt sich einem zunehmenden Anteil kardiovaskulärer und neurologischer Störungen zuordnen. Diese Entwicklung findet sich vor allem in großstädtischen Bereichen wieder, z.B. in Berlin, wo das akute Koronarsyn- 
drom die am häufigsten gestellte notärztliche Diagnose darstellt (5). Diese Tendenz lässt sich auch an anderen Luftrettungsstandorten nachvollziehen $(10,14)$.

Der vermehrte Einsatz von Rettungshubschraubern bei Erkrankungen ist nicht unumstritten, wurde doch der Nutzen des Lufttransportes bei Patienten mit akuten Erkrankungen bereits mehrfach in Frage gestellt. Die Folgen bei Patienten mit Myokardinfarkt und Rhythmusstörungen wurden - mit dem Hinweis auf zusätzliche Stressbelastungen - durch diese Transportform sogar als nachteilig eingestuft (3, 14). Auch wenn der Nutzen der Luftrettung nach wie vor primär an der Versorgung von Traumapatienten und hierbei besonders an Schwerverletzten festgemacht wird $(7,12$, 17), kann diese Entwicklung weder negiert noch ihr Nutzen im Hinblick auf eine frühzeitige stationäre Diagnostik und Therapie bestritten werden.

\section{RTH transportiert immer mehr}

Patienten mit hohem NACA-Index

Die Dokumentation und Vergleichbarkeit von Daten stellt in der präklinischen Notfallmedizin schon sehr lange und häufig ein Problem dar (11, 25). Der NACA-SchweregradIndex wurde zunächst nur zur Beurteilung von Verletzungsintensitäten bei Trauma verwendet, ist aber in der Notfallmedizin inzwischen als Basis zur Objektivierung der Schädigungen etabliert. Im Vergleich zu klinischen Scores (prognostisch-indikativer Aspekt), steht hier der deskriptive Aspekt zur Erfassung von Art und Ausmaß der Schädigung und Bedrohung im Vordergrund (19). Mit dieser Klassifizierung, die seit 1993 für den RTH Ch 18 obligat eingeführt wurde, lassen sich Vergleiche einzelner Standorte, aber auch ganzer Kollektive realisieren (Benchmarking). Der mittlere NACA-Wert bei den Primärpatienten lag bei 3,7 und damit in Übereinstimmung mit anderen RTH-Standorten (8). Über 2/3 der Patienten litten an mittelschweren bis schweren Schädigungen. Dies steht im Gegensatz zum bodengebundenen Rettungsdienst in Bayern, bei dem $78,6 \%$ aller Einsätze

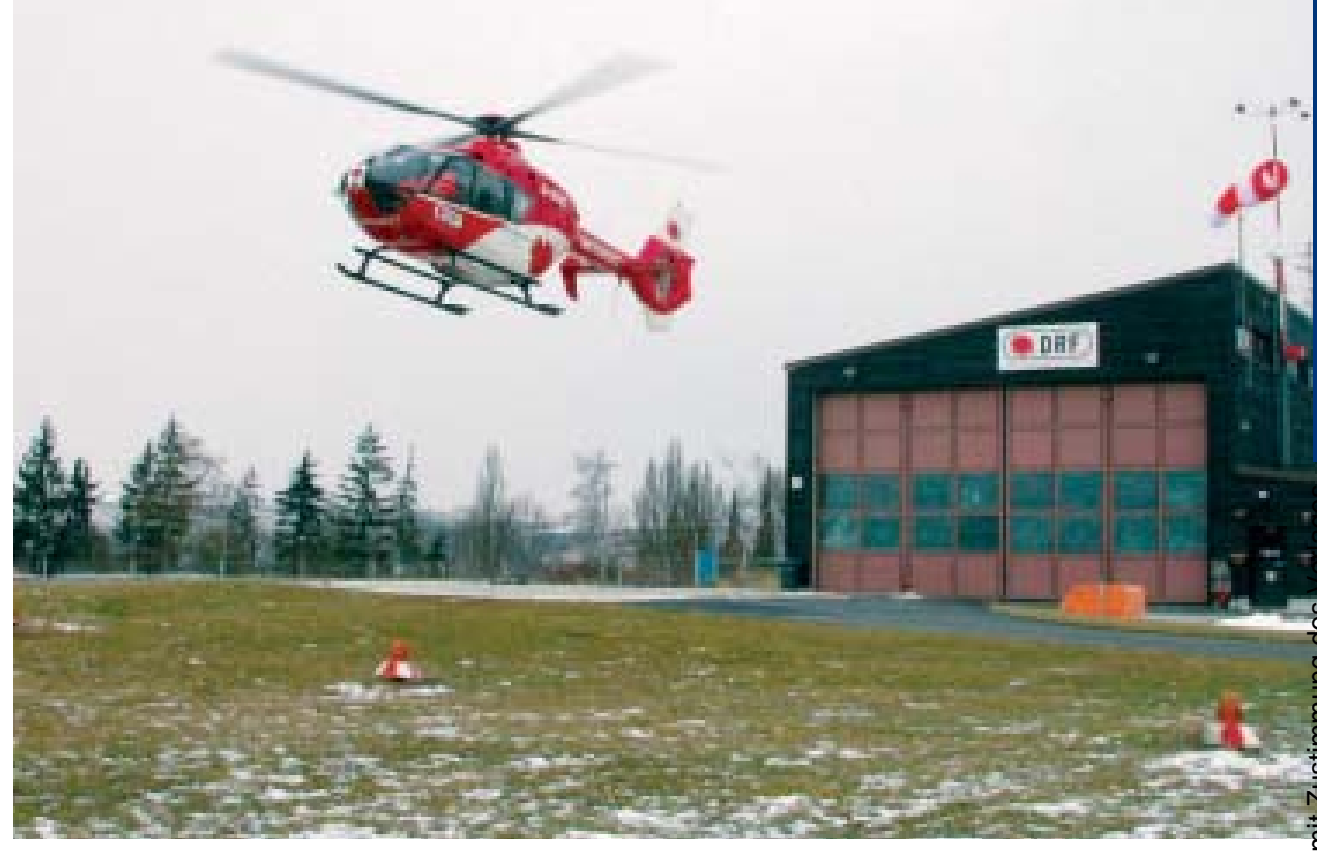

dem NACA-Score I bis III zugeordnet werden müssen (21). Die vergleichbaren Werte III bis V machen 70,2\% aus, woraus deutlich wird, dass der Schwerpunkt der Einsätze des Bodennotarztes beim NACA-Score III liegt (51,9\%). Der RTH führte - als Ausdruck des schwerer geschädigten Patientenkollektives - 3,4\% Reanimationen durch, während im Landrettungsdienst dieser Anteil lediglich $1 \%$ ausmacht. Im Kollektiv des Basler RTH überwiegen die NACA V-Patienten, was durch den hohen Anteil der Trauma-Patienten - speziell Schädel-Hirn-Trauma begründet sein könnte (10). Andererseits wird von den gleichen Autoren eine Notarztindikation erst $a b$ dem NACA-Score IV gesehen, was bestimmt seinen Niederschlag im Dispositionsverhalten der Leitstelle findet. Für den RTH SAR Ulm 71 wurde für den Zeitraum 1980 bis 1991 eine deutliche Zunahme der Patienten mit NACA VI zu Lasten der Patienten mit NACA IV beobachtet, wobei es bei NACA $V$ nur geringe Schwankungen über diese Jahre gab (16). Die jährliche Quersumme (NACA IV + V + VI) blieb dabei konstant. Dies kann generell mit einer Verbesserung der präklinischen Versorgung und verkürzten Rettungszeiten erklärt werden, wodurch Patienten mit komplexeren Krankheitsbildern die Klinik lebend erreichten. Diese Erklärung lässt sich vor allem durch Tabelle 3 veranschaulichen, wobei der Anteil der mit Luftret- tungsmittel transportierten Patienten mit dem steigenden NACA-Wert ansteigt.

\section{Effektivität wird an \\ Reanimationen gemessen}

Nach wie vor wird die Effektivität eines Rettungssystems am Einsatzanlass Reanimation gemessen, da sich hierbei der Erfolg unmittelbar nachweisen lässt. Dies war auch der Grund, weshalb die Reanimation als Einsatzanlass und Therapiemaßnahme gesondert herausgegriffen wurde. Von den im Rahmen des Einsatzes des RTH Ch 18 durchgeführten Reanimationen (bei Primärpatienten) waren $28,5 \%$ primär erfolgreich. Der höchste Anteil erfolgreicher Reanimationen lag zwar bei Patienten mit neurochirurgischen Grundschädigungen - speziell mit Schädel-Hirn-Trauma (66,7\%) - was allerdings durch die kleine Fallzahl nicht repräsentativ sein kann. Die Angaben über Reanimationen im Luftrettungsdienst schwanken zwischen 1,9 und $5,8 \%(8,10,24)$. Dem Luftrettungsdienst darf unterstellt werden, dass weniger Reanimationsversuche unternommen werden als im bodengebundenen Rettungsdienst, was allerdings für den Vergleich RTH Ch 18 (3,4\%) und Gesamtrettungsdienst Bayern nicht zutrifft (3,7\%) (21). Baum errechnete bei einem Anteil von 10\% dauerhaft wiederhergestellten Reanimationspatienten einen Kosten-NutzenQuotienten von 2,2 unter dem 
Aspekt der Kosteneinsparung im Gesundheitswesen. Schon bei 6\% vollständig wiederhergestellten Reanimationspatienten ist die Rentabilitätsschwelle erreicht (6). Obwohl keine Daten bezüglich des weiteren Verlaufs der primär erfolgreichen Reanimation vorliegen, darf bei einer Quote von 28,5\% primär erfolgreicher Reanimationen unterstellt werden, dass die Rentabilitätsmarke überschritten wurde. Allerdings ist bei diesem Patientenkollektiv auffällig, dass die meisten Reanimationsversuche bei internistischen Notfallpatienten mit der höchsten Reanimationsquote von 29,5\% - abgesehen von der geringen Fallzahl der Patienten mit SchädelHirn-Trauma - durchgeführt wurden.

Somit darf eine grundsätzliche Wandlung des Rettungsmittels Hubschrauber in den zurückliegenden Jahren konstatiert werden. Von einem anfänglich auf Verkehrsunfälle und traumatologische Notfälle spezialisierten Rettungsmittel ist der RTH mit seiner Besatzung zu einem universellen präklinischen Versorgungsinstrument geworden. Damit hat sich der RTH in das veränderte Spektrum der Notfallrettung voll integrieren lassen. Und mehr noch: er wurde zu einer Rückversicherung, wenn seitens der Bodenrettung Lücken aufgetreten sind. Die Spezialaufgaben (Zuführung zu Behandlungszentren u.a.) bleiben davon unberührt, sind aber in den Hintergrund getreten. Auf die Luftrettung kann deshalb heute im Rahmen des deutschen Rettungssystems aus medizinischer Sicht nicht verzichtet werden.

\section{Summary}

The value of helicopters in the air rescue service (ARS) within the German medical rescue service continues to prompt discussion. Compared to the earth-bound medical rescue service, the ARS has a number of advantages, which are of particular significance when the site of the emergency is a long distance away. The use of the helicopter is, however, subject to both technical and aeronautical restrictions, so that it must

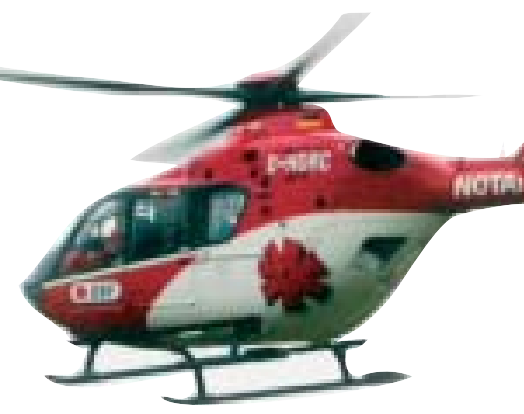

be seen only as a supplementary option. The helicopter and its crew has mutated from a rescue facility initially specialised in road accidents and traumatological emergencies, to a medical rescue option with universal application. As a contribution to further discussions, therefore, the mission data collected by the Christoph 18 rescue helicopter are presented.

\section{Literatur}

1. ADAC-Luftrettung $\mathrm{GmbH}$. Rettungshubschrauberstatistik 1980-1995

2. ADAC-Luftrettung GmbH. Rettungshubschrauber-Information. München: 1992

3. Adamek L, Lenkewitz B, Engelhardt GH. Kriterien der Transportfähigkeit und der Transport schwerverletzter Patienten. Notarzt 1987; 3: 78-89

4. Ahnefeld FW. Bestandsaufnahme Luftrettung. Notfallmed 1993; 19: 280-281

5. Arntz HR, Klatt S, Stern R et al. Sind Notarztdiagnosen zuverlässig? Anaesth 1996; 45: $163-170$

6. Baum H. Wirtschaftliche Erfolgsmessung in der Luftrettung. Notfallmedizinisches Podium 1990: 82-87

7. Baxt WG, Moody P, Cleveland AC et al. Hospital-based rotorcraft aeromedical emergency care service and trauma mortality: A multicenter study. Ann Emerg Med 1985; 14: 61-65

8. Bosch S. Analyse und Evaluierung der Primäreinsätze des Rettungshubschraubers "Christoph 41" bei chirurgischen, internistischen und neurologischen Notfällen. DRFSchriftenreihe, München: Wolfsfellner, 1996 9. Cameron PA, Flott K, Kaan E et al. Helicopter retrieval of primary trauma patients by a paramedic helicopter service. J Surg 1993; 63: 790-797

10. Demartines N, Castelli J, Scheidegger D, Harder F. Entwicklung des HubschrauberRettungskonzeptes in der Region Basel. Schweiz Rundschr, Med Prax 1992; 81: 405-410

11. Dick W. Probleme in der notfallmedizinischen Forschung. Notfallmed. 1991; 17: 538-549

12. Graf M, Demartines N, Harder F, Scheidegger D. Polytrauma: Vergleich des Spitalverlaufes nach Luft- (mit Notarzt) versus Bodentransport (ohne Notarzt). Helv. Chir Acta 1993; 59: 649-653

13. Grundsätze für die Weiterentwicklung der Luftrettung in Deutschland. Abschluß-

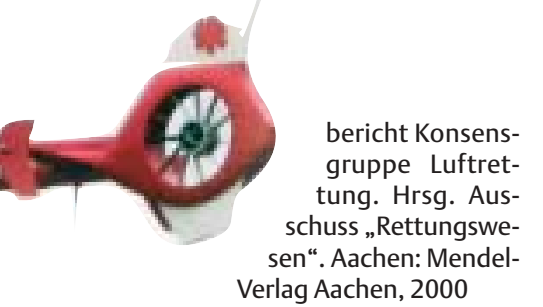

14. Hotred R, Kristiansen JS, Förde $\mathrm{OH}$ et al. Which groups of patient benefit from helicopter evacuation? Lancet 1996; 347: 1362-1366

15. Kugler G. Grundlagen der Luftrettung in der Bundesrepublik Deutschland. Notarzt 1986; 2: 147-149

16. Lampl L, Helm M. Veränderungen im Einsatzspektrum des RTH „SAR 75“-Ein Beitrag zur Effizienzanalyse der Luftrettung. Notarzt 1994; 10: 103-108

17. Nardi G, Massarutti D, Muzzi R et al. Impact of emergency medical helicopter service on mortality for trauma in north-east Italy. Eur J Emerg Med 1994; 1: 69-77

18. Puhan T. Einsatzbedingungen der Luftrettung. In: Luftrettung in der Bundesrepublik Deutschland. Bundesanstalt für Straßenwesen, Bereich Unfallforschung. Bergisch-Gladbach, 1986: 43-55

19. Schuster HP, Dick W. Scoresysteme in der Notfallmedizin? Anaesth. 1994; 43: 30-3

20. Schmiedel R. Leistungen des Rettungsdienstes 1994/95. Berichte der Bundesanstalt für Straßenwesen Heft M 72. Bremerhaven: Verlag für neue Wissenschaft, 1997

21. Sefrin P, Brandt M, Lay A. Der Notarztdienst in Bayern. Bay. Ärztebl. 2001; 56: 313-317

22. Soldner E, Börner M. Statistische Auswertung der Einsätze des Rettungshubschraubers „Christoph 2“ über einen Zeitraum von 10 Jahren. Notarzt 1986; 2: 79-82 23. Sefrin P, Sellner J. Die Bedeutung verschiedener Ausgangsfaktoren für den Krankheitsverlauf von Notfallpatienten. Notarzt 1991; 7: 133-138

24. Stegen H. Reanimationsergebnisse der Deutschen Rettungsflugwacht. In: Luftrettung in der Bundesrepublik Deutschland. Bundesanstalt für Straßenwesen, Bereich Unfallforschung. Bergisch-Gladbach, 1986: 71-97

25. Suakker A, Bernauer J. Dokumentation und statistische Auswertungen von NAWEinsätzen. Notfallmed 1992;18: 540-510

26. Sicherheit im Straßenverkehr. Bericht über Maßnahmen auf dem Gebiet der Unfallverhütung im Straßenverkehr und über das Rettungswesen in den Jahren 1996 und 1997. Bundestagsdrucksache 13/11252 vom 3.7.1998

\section{Anschrift des Verfassers}

Prof. Dr. med. P. Sefrin

Klinik für Anaesthesiologie der Universität Würzburg Sektion für präklinische Notfallmedizin Josef-Schneider-Straße 2

97080 Würzburg 